\title{
Lack of Current Recommendations for Resuming Sexual Activity Following Spinal Surgery
}

\author{
Cara Michelle Thomas ${ }^{1}$, Howard Benjamin Levene ${ }^{2}$ \\ ${ }^{1}$ Department of Physical Medicine and Rehabilitation, Jackson Memorial Hospital, University of Miami, Miami, FL, USA \\ ${ }^{2}$ Department of Neurosurgery, University of Miami, Miami, FL, USA
}

Patients are faced with many questions surrounding the after effects of the various surgical procedures and their ability to return to preoperative activities. While patients often question whether surgery would provide alleviation of pain, weakness, and instability, they often have additional questions about sexual activity during their convalescence that are not always addressed. Although the literature shows postsurgical improvement in sexual activity in association with improved low back pain, reports vaguely address the variability in sexual activity recommendations based on anatomic location and type of spinal surgery. We conducted a PubMed search of the English language from 1990 to 2018 with the following keywords: sexual activity, postoperative, spinal fusion, spinal decompression, functional outcomes, laminectomy, rehabilitation, biomechanics, lumbar disc surgery, metabolic energy expenditure, coital position, and Oswestry Disability Index. Additional studies are needed that survey both patients and spine surgeons to examine current recommendations and to help formulate future guidelines.

Keywords: Sexual behavior; Spine; Safety; Rehabilitation; General surgery

\section{Introduction}

Sexual activity is a fundamental aspect of health and wellbeing for several patients [1]. Despite the importance of sexual health, the topic is often not discussed regularly with patients who suffer from several medical conditions [2]. One medical condition that is common among the elderly is degenerative disease of the spine and the number of spine surgeries performed annually is increasing [3]. The effect of undergoing various types of spinal surgeries on sexual health is common questions among patients. In the literature, the topic of sexual function has been addressed regarding various pathologies, including stroke, cardiac disease, and chronic pain as well as pre- and postoperatively in patients with hip arthritis and diffuse low-grade gliomas [4-7]. However, sexual function in patients who have undergone spinal surgeries has been addressed in a limited number of studies. In a randomized controlled trial by Berg et al. [8], the investigators have studied the effect of total disk replacement (TDR), posterolateral fusion (PLF) or posterolateral interbody fusion (PLIF) on sexual function in patients with chronic lower back pain of discogenic origin. This study included 152 participants who were given gender-specific questionnaires regarding erection, orgasm, and ejaculation. Patients in the treatment groups who underwent TDR and PLF/PLIF procedures reported improvement in their sex lives that was strongly correlated to the reduction in their

Received May 3, 2018; Revised Sep 5, 2018; Accepted Sep 27, 2018

Corresponding author: Howard Benjamin Levene

Department of Neurosurgery, University of Miami, 1475 NW 12th Ave, Miami, FL 33136, USA

Tel: +1-305-243-6946, Fax: +1-305-243-3337, E-mail: HLevene@med.miami.edu 
lower back pain. However, the article did not address the specifics of time to resume sexual activity.

Hagg et al. [9] have studied the effect of anterior fusion surgery for chronic lower back pain on sex-related quality of life. Patients were randomized into non-surgical and surgical treatment groups. Patients who were treated surgically were found to have a better sex life than those who were treated non-surgically, and the improvements in the patient's sex lives were significantly associated with reduction in back pain. In a retrospective cohort study by Horst et al. [10], surgical intervention in patients with degenerative spondylolisthesis and spinal stenosis was to determine the effect of surgery on sexual function. Three operative groups, including surgery without fusion, onelevel fusion, and two (or more)-level fusion, were compared to a non-operative group. Patients received a modified Oswestry Disability Index questionnaire that asked, "In the past week, how has pain affected your sex life?" At all follow-up visits, a significantly lower percentage of patients reported pain in the operative groups with one-level fusion or less compared with that of the non-operative group. Although these studies directly addressed sexual function changes and back pain relief following specific spinal surgeries, such as PLF/PLIF, TDR, and anterior spinal surgeries, the studies did not offer recommendations for resuming sexual activity for postoperative spinal surgery patients.

A PubMed search of the English language literature was performed that used the following keywords: sexual activity, postoperative, spinal fusion, sex life, spinal decompression, functional outcomes, laminectomy, rehabilitation, biomechanics, lumbar disk surgery, metabolic energy expenditure, metabolic equivalent, coital position, and Oswestry Disability Index. The literature review spanned the dates of 1990-2018. From this search, 27 articles were reviewed and 21 were found to be relevant.

\section{Patient Case}

During an outpatient neurosurgery clinic, a 51-year-old male patient presented with a 1-year history of progressive electric-type pain in his upper extremities with associated paresthesias and allodynia. He reported no identifiable precipitating factors, including trauma or falls. Cervical magnetic resonance imaging revealed a cervical disk bulge at $\mathrm{C} 5-\mathrm{C} 6$ with myelomalacia at the adjacent spinal cord level. Upon discussion of treatment options, the patient opted to proceed with an anterior cervical discectomy and fusion from $\mathrm{C} 5$ to $\mathrm{C} 6$. After discussing the risks and benefits of surgery with his life partner, the patient also had questions about when it would be safe to resume sexual activity, particularly oral sex, after surgery. Ideally, the spine surgeon would be able to guide the patient on subsequent activities based both on the specific surgery and the specific sexual activity. Different types of spinal surgeries would be associated with different physical constraints. Indeed, a microdiscectomy has a very different physical effect than that of a multilevel scoliosis deformity surgery. Moreover, the physical level of sexual activity can vary considerably depending on the preference of the partner or partners. Unfortunately, no direct guidance exists for any of these categories. Any answer given to this patient and his partner amounts to the physician's best guess but would not be directly supported by literature evidence.

\section{Discussion}

There is an association between the quality of a patient's sex life and the degree of lower back pain. In a questionnaire-based study by Maigne and Chatellier [11], patients with low back pain reported a greater interference in sexual activity compared with that reported by patients with neck pain. The biomechanics of sexual positions is a very interesting topic, and to the best of our knowledge, literature that discusses specific recommendations for postoperative spinal movements during coitus or other sexual interactions is lacking. A textbook by White and Panjabi [12] published in 1990 recommends side-lying positions as the "best basic position for either partner with low back pain." This recommendation was based on the reasoning that hip and knee flexion would relax the psoas and sciatic nerve, thereby straightening the spine and reducing a disc bulge. Although this recommendation by White and Panjabi [12] is a logical extrapolation, it was not directly examined until later studies that measured the biomechanics of the spine during coitus. Sidorkewicz and McGill [13] have measured and discussed the biomechanical stresses of spinal movement and positions during coitus to develop recommendations for male patients with low back pain. This study included a group of 10 healthy male and female participants engaged in various sexual positions, including quadruped, missionary, and side-lying. The lumbar spine, torso, and pelvic kinematics were measured using an optoelectronic motion capture system that 
measured the lumbar spine angles during sexual activity. The study demonstrated a variation in the male spine depending on the coital position; however, the majority of the range was in flexion for all positions. On the basis of these results, coital position recommendations were made that were based on specific movements that exacerbated lower back pain in individual participants. For example, flexion-intolerant participants were advised to avoid sidelying and missionary (male supporting upper body with elbows) positions. Conversely, extension-intolerant participants were advised to avoid quadruped and missionary (male supporting upper body with extended arms) positions. Sidorkewicz and McGill [14] have conducted an additional study that documented female spine motion during coitus with commentary on associated low back pain. The study concluded that side-lying was beneficial for women who were motion-intolerant. Interestingly, the earlier study had not arrived at the same conclusion for men; there were no ideal positions for men who were motion-intolerant. The coital positions for women can vary from more extension to more flexion than those for men, whereas men are mostly in a flexed position during coitus. These studies were in healthy volunteers and assessed the lumbar spine without including cervical spine positions or postoperative patients; however, these studies suggested that coital positions have unique alterations to spinal biomechanics and could be used to develop restrictions depending on the pain and mechanical stresses felt by patients healing from surgery.

Although mechanical stresses on the spine during sexual activity are definitely a concern for spine professionals, there may be additional considerations with respect to postoperative sexual activity recommendations, such as energy expenditure, anatomical location, and type of surgery.

Following neurosurgical intervention, comprehensive rehabilitation is commonly recommended for patients to improve functionality. In a randomized controlled trial by Mannion et al. [15], patients who had undergone decompression surgery for spinal stenosis and had received physiotherapy showed a significant reduction in selfrated disability, pain intensity/frequency, and psychological disturbance compared with those who received no formal physiotherapy postoperatively. Ostelo et al. [16] performed a systematic review of 13 studies to assess the effectiveness of rehabilitative treatments following a firsttime lumbar disc surgery and found strong evidence that intense exercise programs initiated 4-6 weeks postoperatively were more effective on functional status and allowed faster return to work than did mild exercise programs.

Given the lack of data, spine surgeons and rehabilitation specialists must attempt to extrapolate guidelines from a mixture of their own clinical experience and current postsurgical exercise guidelines. Considering sexual activity as a type of exercise, sexual activity can be classified as being in the moderate intensity range for energy expenditure and as significant as traditional exercise [17]. In a study by Frappier et al. [17], 21 young heterosexual couples completed an endurance exercise session using armbands to measure energy expenditure. The couples also performed sexual activities once a week for 4 weeks and wore the energy expenditure armband during sexual activity. The results of the study showed that the mean energy expenditure during sexual activity and the endurance sessions was 6.0 metabolic equivalents (METS) and 8.5 METS, respectively, in men and 5.6 METS and 8.4 METs, respectively, in women,. Similar recreational and household activities that fall into the moderate level of exercise intensity include gardening, aerobic dancing, brisk walking, tennis (doubles), and bicycling (at $<15 \mathrm{~km} / \mathrm{hr}$ ) [18]. Assuming that sexual activity is considered a moderate intensity activity and intense exercise programs are recommended at 4-6 weeks postoperatively after a microdiscectomy, then it can be extrapolated that resuming sexual activity at this time is safe. Despite the several assumptions made here, this recommendation would involve only one kind of spinal surgery, the microdiscectomy.

\section{Conclusions}

Despite the frequency and importance of this topic, recommendations regarding the time to resume sexual activity following any type of spinal surgery have not been established among spine surgeons and rehabilitation specialists. Moreover, recommendations on safe sexual positions following spinal surgery are elusive in the literature. Patients who have had similar procedures may be given markedly different timelines for resuming sexual activity at the discretion of their individual surgeon. In several cases, patients may not be provided any guidance and are forced to rely on their level of postsurgical pain as a guide for resuming sexual activity. Because of the importance of discussing sexual function with patients, more specific guidelines regarding timelines for safely resuming sexual 
activity postoperatively are needed. The complexities of the spinal interventions, breadth of the surgeries, pain levels, and physical demands of various coital/sexual positions are all vital components that must be considered when proposing standardized guidelines. To access the clinical experience of spine surgeons, we propose future studies that survey current recommendations from the spine community, which would be a vital first step toward formulating standardized patient recommendations for postoperative sexual activity. We will also plan to generate surveys for postoperative spinal surgery patients to discover the actual time course of return to their normal sexual activity. Although the topic may be taboo to some, it remains the responsibility of spine surgeons and rehabilitation specialists to provide appropriate guidance to their patients in all physical activities, including sexual, throughout their recovery.

\section{Conflict of Interest}

No potential conflict of interest relevant to this article was reported. This narrative review was not presented at an American Academy of Physical Medicine and Rehabilitation Annual Assembly. We did not use any medical devices in this literature review. There was no funding provided to us for this study.

\section{References}

1. Kreuter M, Taft C, Siosteen A, Biering-Sorensen F. Women's sexual functioning and sex life after spinal cord injury. Spinal Cord 2011;49:154-60.

2. Ng L, Sansom J, Zhang N, Amatya B, Khan F. Effectiveness of a structured sexual rehabilitation programme following stroke: a randomized controlled trial. J Rehabil Med 2017;49:333-40.

3. Williams MG, Wafai AM, Podmore MD. Functional outcomes of laminectomy and laminotomy for the surgical management lumbar spine stenosis. J Spine Surg 2017;3:580-6.

4. Surbeck W, Herbet G, Duffau H. Sexuality after surgery for diffuse low-grade glioma. Neuro Oncol 2015;17:574-9.

5. Laffosse JM, Tricoire JL, Chiron P, Puget J. Sexual function before and after primary total hip arthroplasty. Joint Bone Spine 2008;75:189-94.

6. Steinke E, Patterson-Midgley P. Sexual counseling following acute myocardial infarction. Clin Nurs Res 1996;5:462-72.

7. Ambler N, Williams AC, Hill P, Gunary R, Cratchley G. Sexual difficulties of chronic pain patients. Clin J Pain 2001;17:138-45.

8. Berg S, Fritzell P, Tropp H. Sex life and sexual function in men and women before and after total disc replacement compared with posterior lumbar fusion. Spine J 2009;9:987-94.

9. Hagg O, Fritzell P, Nordwall A; Swedish Lumbar Spine Study Group. Sexual function in men and women after anterior surgery for chronic low back pain. Eur Spine J 2006;15:677-82.

10. Horst PK, Khanna K, Racine L, et al. Sex life and impact of operative intervention on sex life-related pain in degenerative spinal conditions: an analysis of the sport study. Spine 2016;41:1764-71.

11. Maigne JY, Chatellier G. Assessment of sexual activity in patients with back pain compared with patients with neck pain. Clin Orthop Relat Res 2001:82-7.

12. White AA, Panjabi MM. Clinical biomechanics of the spine. 2nd ed. Philadelphia (PA): Lippincott Company; 1990.

13. Sidorkewicz N, McGill SM. Male spine motion during coitus: implications for the low back pain patient. Spine 2014;39:1633-9.

14. Sidorkewicz N, McGill SM. Documenting female spine motion during coitus with a commentary on the implications for the low back pain patient. Eur Spine J 2015;24:513-20.

15. Mannion AF, Denzler R, Dvorak J, Muntener M, Grob D. A randomised controlled trial of post-operative rehabilitation after surgical decompression of the lumbar spine. Eur Spine J 2007;16:1101-17.

16. Ostelo RW, de Vet HC, Waddell G, Kerckhoffs MR, Leffers P, van Tulder MW. Rehabilitation after lumbar disc surgery. Cochrane Database Syst Rev 2002:CD003007.

17. Frappier J, Toupin I, Levy JJ, Aubertin-Leheudre $\mathrm{M}$, Karelis AD. Energy expenditure during sexual activity in young healthy couples. PLoS One 2013;8:e79342.

18. Jette M, Sidney K, Blumchen G. Metabolic equivalents (METS) in exercise testing, exercise prescription, and evaluation of functional capacity. Clin Cardiol 1990;13:555-65. 